Published in final edited form as:

Am J Obstet Gynecol. 2008 May ; 198(5): 570.e1-570.e5.

\title{
Changes in Physical Activity after Abdominal Sacrocolpopexy for Advanced Pelvic Organ Prolapse
}

\author{
Ingrid Nygaarda ${ }^{\mathrm{a}}$, Victoria L Handa ${ }^{\mathrm{b}}$, Linda Brubakerc, Diane Borello-France ${ }^{\mathrm{d}}$, John Wei ${ }^{\mathrm{e}}$, \\ Ellen Wells ${ }^{f}$, and Patricia Goode ${ }^{g}$ for the Pelvic Floor Disorders Network

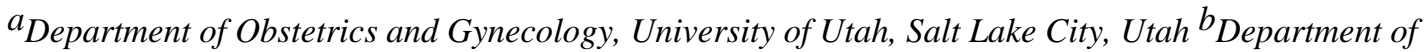 \\ Gynecology and Obstetrics, Johns Hopkins University, Baltimore, Maryland ${ }^{c}$ Departments of Obstetrics \& \\ Gynecology and Urology, Loyola University Medical Center, Maywood, Illinois $d_{\text {Department of Physical }}$ \\ Therapy, Duquesne University, Pittsburgh, PA e Department of Urology, University of Michigan, Ann Arbor, \\ Michigan $f_{\text {Department of Obstetrics and Gynecology, University of North Carolina at Chapel Hill, Chapel }}$ \\ Hill, North Carolina ${ }^{g}$ Birmingham/Atlanta Geriatric Research, Education, and Clinical Center, Veterans \\ Affairs Medical Center, Birmingham, AL and Division of Gerontology, Geriatrics and Palliative Care, \\ University of Alabama at Birmingham, Birmingham, $A L$
}

\begin{abstract}
Objective-To describe changes in physical activity one year after sacrocolpopexy for pelvic organ prolapse.

Study design-Prospective cohort of 301 randomized trial participants.

Results-Compared to baseline, one year after surgery, 36\% increased, $18 \%$ decreased and $47 \%$ did not change pre-operative exercise intensity level. In contrast, women were more likely to reduce (24\%) than increase (11\%) the frequency of major effort activities, like heavy lifting. Of 99 women who reported pre-operatively that prolapse interfered substantially with doing exercise or recreation, house/yardwork, or work outside the home, 83 (84\%) reported no substantial interference one year later. After surgery, women reporting substantial interference from prolapse or treatment had similar rates of interval treatment for stress incontinence or prolapse as women not reporting substantial interference.
\end{abstract}

Conclusion-After sacrocolpopexy, one-third of women increased exercise intensity, few increased major effort activities and most reported that prolapse no longer interfered with activities.

Condensation: Women are more likely to engage in a higher level of exercise after sacrocolpopexy but are less likely to perform physical activities requiring a major effort such as lifting or straining.

\section{Keywords}

pelvic organ prolapse; sacrocolpopexy; physical activity; exercise

Corresponding author: Ingrid Nygaard, MD, Department of Obstetrics and Gynecology, University of Utah College of Medicine, 30 North 1900 East, Room 2B 242, Salt Lake City, UT 84132, Tel: 801-581-5490, Fax: 801- 585-5146, E-mail address: Ingrid.nygaard@hsc.utah.edu.

Presented at the American Urogynecologic Society in Hollywood, Florida September, 2007.

Publisher's Disclaimer: This is a PDF file of an unedited manuscript that has been accepted for publication. As a service to our customers we are providing this early version of the manuscript. The manuscript will undergo copyediting, typesetting, and review of the resulting proof before it is published in its final citable form. Please note that during the production process errors may be discovered which could affect the content, and all legal disclaimers that apply to the journal pertain. 


\section{INTRODUCTION}

There are scant data about the impact of pelvic organ prolapse, or its treatment, on physical activity. We previously reported that the frequency of mild, moderate, or strenuous exercise did not differ by prolapse stage in women with pelvic organ prolapse planning sacrocolpopexy but who did not have symptoms of stress incontinence. ${ }^{1}$ However, $27 \%, 19 \%$, and $8 \%$ of women stated pre-operatively that prolapse substantially interfered with exercise or recreation, housework or yard work, and work outside the home, respectively. Compared to women with less symptom distress, women with greater symptom distress were more likely to report that prolapse interfered with household or yard work, recreation, and employment. Since regular physical activity has many benefits, including improved psychological well-being, lower death rates, and lower risk of developing coronary heart disease, stroke, hypertension, and diabetes $^{2}$, it is important to ascertain if repair of pelvic organ prolapse results in increased physical activity.

The aims of this prospective study of women one year after sacrocolpopexy for pelvic organ prolapse are to 1) determine the change from baseline in the proportion of women who report participation in recreational exercise and strenuous physical activities, 2) determine the postoperative change in interference from either prolapse or its treatment with ability to do housework, work outside the home, or participate in exercise or recreation, and 3) to describe factors that contribute to current interference with physical activity, such as physician instructions, fear of prolapse recurrence, recurrent prolapse urinary leakage, or other medical problems.

\section{Methods}

Participants included women who enrolled in and completed one-year follow-up in "Colpopexy And Urinary Reduction Efforts" (CARE), a randomized trial designed to evaluate whether a standardized modified Burch colposuspension, when added to abdominal sacrocolpopexy to treat POP, improves urinary stress continence in subjects without preoperative symptoms of stress urinary incontinence. ${ }^{3}$ The CARE trial was performed through the Pelvic Floor Disorders Network (PFDN) sponsored by the National Institute of Child Health and Human Development (one of the National Institutes of Health). Each clinical site, subsites, and the Data Coordinating Center received Institutional Review Board approval for this trial and all subjects provided written informed consent.

Participants completed a standardized assessment before surgery and one year later that included validated instruments to assess pelvic symptoms and severity (Pelvic Floor Distress Inventory, PFDI) ${ }^{4}$ condition-specific life impact (Pelvic Floor Impact Questionnaire, PFIQ), and general health-related quality of life (SF-36). ${ }^{5}$ Pelvic organ prolapse was assessed using the POP-Q system ${ }^{6}$ with subjects in lithotomy position during maximal Valsalva effort.

To assess physical activity, we asked questions related to work, and household and recreational activities (Table 1). The item regarding the impact of working outside the home (question 3, Table 1) was addressed only to women who reported such work. To assess current exercise level, we used a modified version of the Godin Leisure-Time Exercise Questionnaire ${ }^{7}$ (Question 1, Table 1), which asked women how often during the last three months, they regularly did strenuous, moderate, or mild exercise. Specific activity examples were provided for each exercise level. Responses were not restricted to one exercise category. We dichotomized a priori the response to major effort activities (question 5 in Table 1) based on a frequency of at least 2-3 times per month (versus less frequently). We defined substantial interference from prolapse when women reported that prolapse interfered "most/all of the time" with activity or that they were "no longer able to do these activities." 
Fisher's exact test was used to compare proportions. Changes between baseline and 12 months were assessed by McNemar's test. A p $<0.05$ was considered significant.

\section{RESULTS}

Of 322 women enrolled in the CARE trial, the 301 that completed data collection both at baseline and at one year are included in this ancillary study. At baseline, 39 women (13\%) had Stage II, 203 (67\%) Stage III, and 59 (20\%) Stage IV POP. There were 13\% below age 50, $26 \%$ between 50 and 59 years, 38\% between 60 and 69 years, and $23 \% 70$ years or greater. Education level was high school or less in $139(46 \%)$ of women. One hundred nineteen women $(40 \%)$ reported surgery for prolapse or incontinence before the sacrocolpopexy.

One year after surgery, women were more likely to report a higher level of exercise $(\mathrm{p}<0.001)$; $107(36 \%)$ increased their pre-operative exercise intensity, while $54(18 \%)$ decreased and 140 women (47\%) were unchanged. (Table 2) A minority (16\%) participated in strenuous activity both before and after surgery.

In contrast to the observed shift toward increased recreational exercise levels one year after surgery, women were more likely to reduce than increase the frequency of major effort activities after surgery: $72(24 \%)$ and $33(11 \%)$, respectively $(\mathrm{p}<0.0001)$. The remaining 196 women (65\%) maintained their baseline frequency of major effort activity: $130(43 \%)<2$ or 3 times per month and $66(22 \%)$ more frequently.

Of the 99 women who reported before surgery that prolapse interfered substantially with their ability to do exercise or recreation, house/yardwork, or work outside the home, $83(84 \%)$ reported no interference one year later. (Figure 1, Table 3) Only 16 of the 202 (8\%) who did not report substantial interference with activities before surgery did report substantial interference with at least one activity at follow-up. Six of these either reported SUI symptoms at one year or had undergone treatment for SUI after the index surgery; this rate of SUI prevalence or retreatment was no different from the rate (35\%) for women who did not report substantial interference at 12 months $(\mathrm{p}=0.78)$. Fourteen of the 16 women reporting de novo substantial interference at 12 months had POP-Q data at one year. There was no association between new onset of substantial interference with activities at one year and post-operative vaginal support; 1 (7.1\%), $8(57.1 \%), 4(28.6 \%)$, and 1 (7.1\%) women that reported new onset substantial interference were stages $0,1,2$, and 3 one year after surgery, respectively, compared to $41(17.2 \%), 113(47.1 \%), 83(34.6 \%)$ and $3(1.3 \%)$ women who did not have substantial interference at one year ( $\mathrm{p}=1.0$ comparing stages 2 and 3 vs 0 and 1$)$.

Similarly, 16 women who reported substantial interference with activities both at baseline and at one year were not more likely to have interval SUI treatment, SUI symptoms at one year, or more advanced prolapse at one year.

Reasons that women provided for why their condition interfered with activities one year after surgery are shown in Table 4 . Most of these women indicated that their prolapse or treatment interfered with activity performance only "some of the time", and interfered more often during exercise/recreation and housework/yardwork than employment. Physician advice to restrict activities and other medical conditions were the most frequent responses accounting for activity interference.

\section{DISCUSSION}

Sacrocolpopexy favorably affected physical activity one year after surgery. One-third of the women in this study reported a higher level of exercise one year after surgical treatment of pelvic organ prolapse. Presumably, these women increased their exercise level in response to 
a reduced prolapse-related symptoms and activity interference. Given the physical, mental, and psychological health burden of a sedentary lifestyle ${ }^{8,9}$, we believe this to be an important outcome of surgery for severe prolapse.

Although we observed a shift from women reporting no exercise/mild exercise to moderate/ strenuous exercise following surgery, in this study, we were unable to determine if women were exercising sufficiently for maximum health benefits. The Healthy People 2010 objectives for physical activity in adults indicate that moderate or vigorous (at 70\% of maximum heart rate) physical activity for at least 30 minutes should be encouraged as part of a daily routine. 10 Recreational activities, yardwork/housework, and employment are of course important and have great potential to impact quality of life, but resuming these activities may not have the same health impact as exercise. For example, in a large study of men and women 70-79 years old, those who participated in 20-30 minutes of moderately intense exercise on most days of the week had better physical function than those who were active throughout the day or who were inactive. ${ }^{11}$ To discern the full health benefits of exercise, physicians should query the patient regarding exercise frequency. Likewise, patients should be advised by their physician or another health care professional with expertise in exercise prescription (physical therapist or athletic trainer) on how to gradually and safely implement their exercise program and on how to monitor their physiological responses to exercise.

While most women in this study reported less interference from prolapse after surgery, some had ongoing or new interference from prolapse or its treatment one year after surgery. Of these, about half did not report interference at baseline. At one year follow-up, women who reported "substantial" interference were not more likely to have symptoms of stress incontinence or recurrent prolapse at one year, or to have required interval treatment of stress incontinence, than women that did not report substantial interference at follow-up. Thus, it does not appear that interference reported by these participants after surgery is related to prolapse or incontinence symptoms.

One year after surgery, a common reason cited for interference with physical activity was physician advice ("my doctor advised me not to do these activities"). We believe that such advice is based on expert opinion rather than scientific evidence, as there is currently insufficient scientific evidence to suggest an adverse effect of physical activity one year after surgery for prolapse. Indeed, in one study, many activities commonly restricted following surgery for pelvic floor disorders did not increase intra-abdominal pressure more than simply rising from a chair. ${ }^{12}$

Women also cited a fear of prolapse recurrence as a reason for interference with activities at one year ("I'm afraid my condition will recur if I do them"). Given the known health benefits of regular physical exercise, it is not clear whether the potential risks of recurrence outweigh the benefits of physical activity in this setting. This is an important area for future research. In the meantime, patients who desire to gain an increase in physical activity or exercise level but are fearful to do so might benefit from referral to a physical therapist for exercise instruction.

A limitation of this research is that treatment protocols and patient instructions were not standardized across surgeons. Thus, variations in physician advice regarding physical activities could affect our results. Also, activities were self-reported by the participants and some underor overestimation of physical activities might have occurred. The instrument we used to measure recreational activity was validated in a group of educated white collar workers and may be less reliable in our population. In addition, we did not have a non-intervention (control) group and it is conceivable that without intervention, physical activity would have declined further in this population. Therefore our findings may underestimate the impact of surgery on physical activity in women with symptomatic prolapse. An additional limitation of this work 
is that some subgroups were small (for example, the number of women with new onset of substantial interference with activities), and statistical inferences drawn may be incorrect. Finally, our results may be specific to the surgical intervention studied in this research (sacrocolpopexy with or without Burch urethropexy) and may not be generalizable to other surgeries or to nonsurgical treatment of prolapse.

In conclusion, one year after abdominal sacrocolpopexy, women are more likely to increase exercise intensity and far less likely to report perceived interference with physical activity due to prolapse or its treatment. Most women do not restrict activities one year later but of the minority that do, many report that they are following physicians' advice.

\section{Acknowledgements}

Supported by grants from the National Institute of Child Health and Human Development and Office of Research in Women's Health (U01 HD41249, U10 HD41268, U10 HD41248, U10 HD41250, U10 HD41261, U10 HD41263, U10 HD41269, and U10 HD41267).

\section{References}

1. Nygaard I, Handa V, Brubaker L, Borello-France D, Wei J, Wells E, Weber AM. for the Pelvic Floor Disorders Network. Physical activity in women planning sacrocolpopexy. Int Urogynecol J 2007;18 (1):33-37.

2. U.S. Department of Health and Human Services. Physical Activity and Health: A Report of the Surgeon General. Atlanta, GA: Centers for Disease Control and Prevention (CDC), National Center for Chronic Disease Prevention and Health Promotion; 1996.

3. Brubaker L, Cundiff G, Fine P, Nygaard I, Richter H, Visco A, Zyczynski H, Brown MB, Weber A. A randomized trial of colpopexy and urinary reduction efforts (CARE): design and methods. Controlled Clinical Trials 2003;24:629-42. [PubMed: 14500059]

4. Barber MD, Kuchibhatla M, Pieper CF, Bump RC. Psychometric evaluation of 2 comprehensive condition-specific quality of life instruments for women with pelvic floor disorders. Amer J Obstet Gynecol 2001;185:1388-1395. [PubMed: 11744914]

5. Ware JE Jr, Gandek B. Overview of the SF-36 health survey and the international quality of life assessment (IQOLA) project. J Clin Epidemiol 1998;51:903-912. [PubMed: 9817107]

6. Bump RC, Mattiasson A, Bo K, et al. The standardization of terminology of female pelvic organ prolapse and pelvic floor dysfunction. Am J Obstet Gynecol 1996;175:10-17. [PubMed: 8694033]

7. Godin G, Shepherd RJ. A simple method to assess exercise behavior in the community. Can J Appl Sport Sci 1985;10:141-6. [PubMed: 4053261]

8. Yaffe K, Barnes D, Nevitt M, et al. A prospective study of physical activity and cognitive decline in elderly women. Arch Intern Med 2001;161:1703-1708. [PubMed: 11485502]

9. Kushi LH, Fee RM, Folsom AR, Mink PJ, Anderson KE, Sellers TA. Physical activity and mortality in postmenopausal women. JAMA 1997;277:1287-1292. [PubMed: 9109466]

10. U.S. Department of Health and Human Services Healthy People 2010: With Understanding and Improving Health. Objectives for Improving Health. 2. 2. 174. U.S. Government Printing Office; Washington, DC: 2006 Mar 14. p. 801-9.Warburton CMAJ

11. Brach JS, Simonsick EM, Kritchevesky S, Yaffe K, Newman AB. for the Health. Aging and Body Composition Study Research Group. JAGD 2004;52:502-509.

12. Weir LF, Nygaard IE, Wilken J, Brandt D, Janz KF. Postoperative Activity Restrictions: Any Evidence? Obstet Gynecol 2006;107:305-309. [PubMed: 16449116] 


\section{Percent of Women with Substantial Interference from POP}

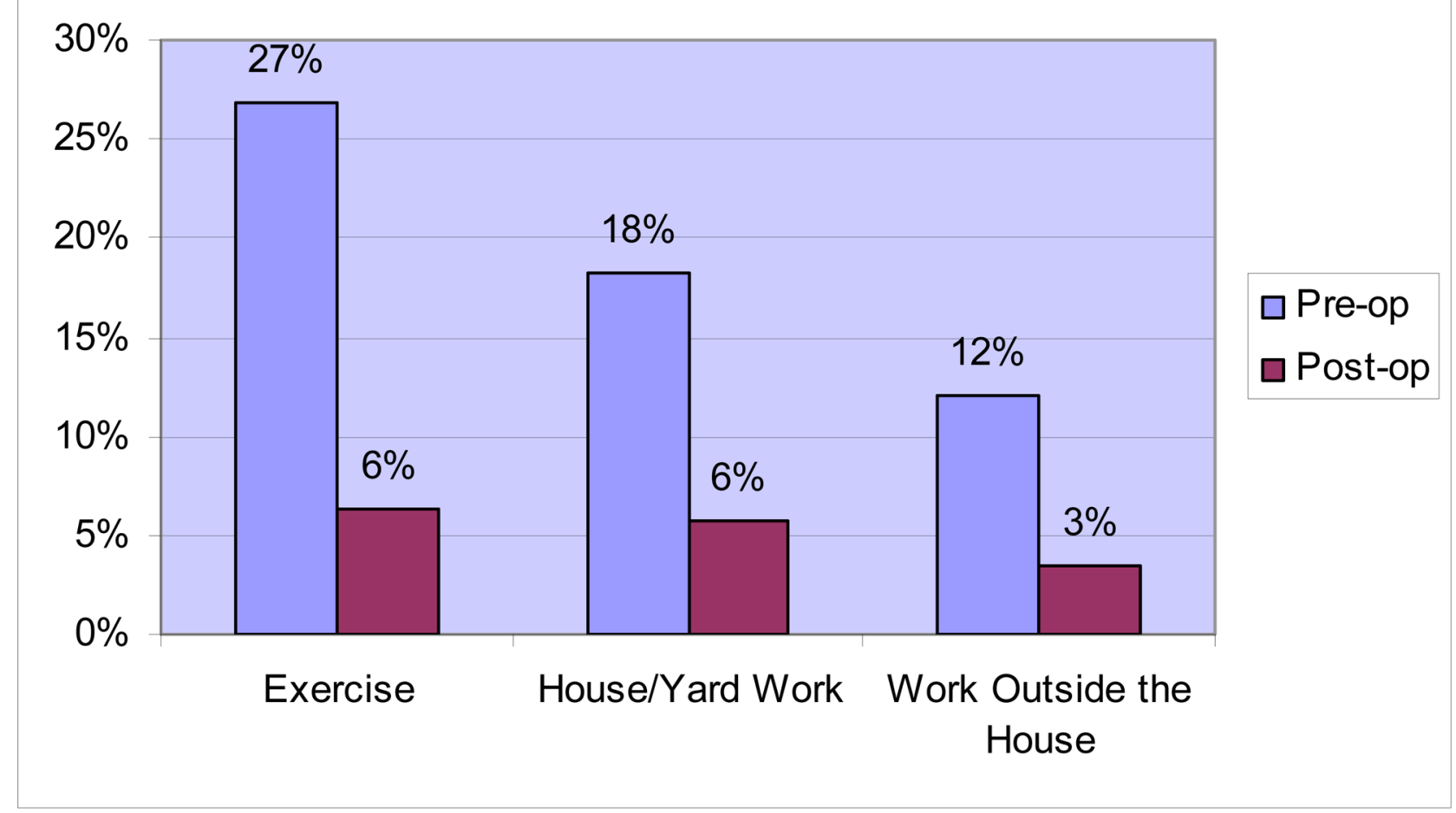

Figure 1. 


\section{PHYSICAL ACTIVITY QUESTIONS}

\section{TABLE 1}

1 During the last three months, on average, how often each week do you usually do the exercises below (state number of days per week):

a. Strenuous or very hard exercise (you work up a sweat and your heart beats fast). For example, aerobics, dancing, jogging, or tennis.

b. Moderate exercise (not exhausting). For example, biking, using an exercise machine, easily swimming, brisk walking.

c. Mild exercise. For example, bowling, golf, walking slowly.

2 During the past three months, has your pelvic prolapse or its treatment interfered with your ability to do housework or yard work? Response categories for questions $2-4$
a. None of the time
b. Some of the time
c. Most/all of the time
d. No longer do these activities

2A. Which one of the following contributed to this reason?

Response categories for questions $2 \mathrm{~A}-4 \mathrm{~A}$ :

a. My doctor advised me not to do these activities

b. I am afraid my condition will recur if I do them

c. Leakage of urine keeps me from doing them

d. Recurrent prolapse keeps me from doing them

e. Other medical problems keep me from doing them

3 During the last three months, has your pelvic prolapse or its treatment interfered with your ability to perform your job outside the home the way you would like?

3A. Which one of the following contributed to this reason?

4 During the last three months, has your pelvic prolapse or its treatment interfered with your ability to participate in the exercise or recreation that you would like to?

4A. Which one of the following contributed to this reason?

5 During the past three months, how often did you perform physical activities that required a major effort, such as lifting heavy furniture, shoveling snow, or lifting people or objects weighing more than $25 \mathrm{lbs}$ ?
a. Never
b. Less than once per month
c. Once per month
d. 2-3 times per month
e. Once per week
f. More than once per week 
Table 2

Change in exercise activity 1 year after surgery

\begin{tabular}{|l|c|c|c|c|}
\hline \multicolumn{4}{|c|}{ Activity level 1 year after surgery; $\boldsymbol{n}(\boldsymbol{\%})^{*}$} \\
\hline Baseline activity level & No exercise & Mild & Moderate & Strenuous \\
\hline No exercise & $12(30)$ & $13(33)$ & $9(23)$ & $6(15)$ \\
\hline Mild & $6(9)$ & $20(29)$ & $27(39)$ & $17(24)$ \\
\hline Moderate & $2(2)$ & $13(12)$ & $61(55)$ & $35(32)$ \\
\hline Strenuous & $2(3)$ & $8(10)$ & $23(29)$ & $47(59)$ \\
\hline
\end{tabular}

Row totals may exceed $100 \%$ due to rounding. 
Table 3

CHANGE IN SUBSTANTIAL INTERFERENCE*

Substantial interference at one year

\begin{tabular}{lll}
\hline Substantial interference at baseline & Yes & No \\
\hline Housework/yardwork & $\begin{array}{l}7(13 \%) \\
10(4 \%)\end{array}$ & $48(87 \%)$ \\
Yes: 55 & $236(96 \%)$ \\
No: 246 & $8(10 \%)$ & $73(90 \%)$ \\
\hline Exercise/recreation & $11(5 \%)$ & $209(95 \%)$ \\
Yes: 81 & $2(10 \%)$ & $19(90 \%)$ \\
No: 220 & $4(3 \%)$ & $150(97 \%)$ \\
\hline Work outside home & $16(16 \%)$ & $83(84 \%)$ \\
Yes: 21 & $16(8 \%)$ & $186(92 \%)$ \\
No: 154 & & \\
\hline Any of the above & & \\
Yes: 99 & & \\
No: 202 & &
\end{tabular}




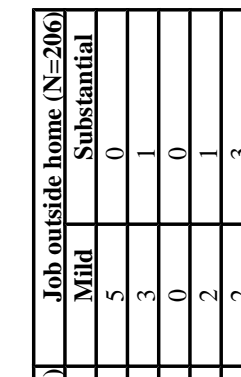

(2)

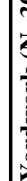

畒

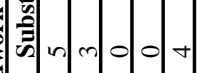

政

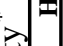
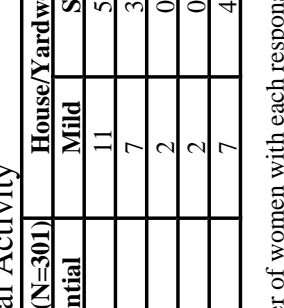

:
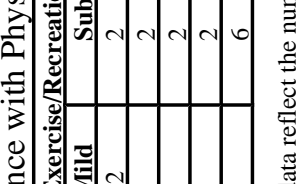

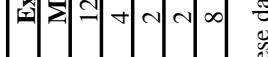

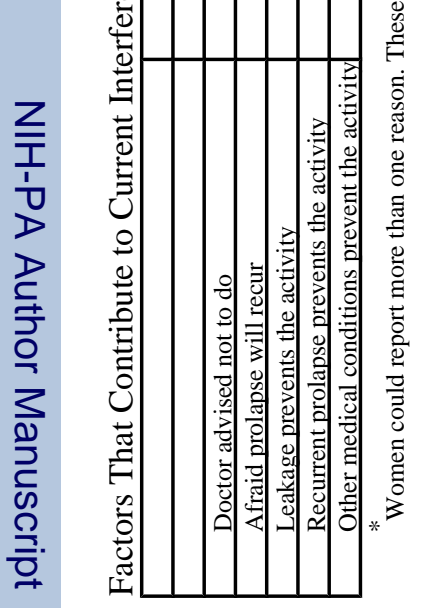

